\title{
Demographics and Hospital Outcomes in American Women With Endometriosis and Psychiatric Comorbidities
}

Chris A. Robert ${ }^{1}$, Emmanuelle J. Caraballo-Rivera ${ }^{2}$, Sasank Isola ${ }^{3}$, Kosisochukwu Oraka ${ }^{4}$, Sabiha Akter ${ }^{5}$, Shikha Verma ${ }^{6,7}$, Rikinkumar S. Patel ${ }^{8}$

1. Obstetrics and Gynecology, Sunrise Hospital, Pune, IND 2. Medicine, Ross University School of Medicine, Bridgetown, BRB 3. Medicine, American University of the Caribbean School of Medicine, Cupecoy, SXM 4. Medicine, Vinnytsia National Medical University, N.I Pirogov, Vinnytsia, UKR 5. Psychiatry, Bergen New Bridge Medical Center, Paramus, USA 6. Psychiatry and Behavioral Health, Rosalind Franklin University, North Chicago, USA 7. Child and Adolescent Psychiatry, Rogers Behavioral Health, Kenosha, USA 8. Psychiatry, Griffin Memorial Hospital, Norman, USA

Corresponding author: Rikinkumar S. Patel, rpatel_09@arcadia.edu

\section{Abstract \\ Objectives}

To explore sociodemographic differences and hospital outcomes in endometriosis patients with versus without psychiatric comorbidities.

\section{Methods}

We conducted a cross-sectional study using the Nationwide Inpatient Sample (NIS, 2012-2014), and included 63,160 females with primary diagnosis of endometriosis. We used descriptive statistics and Pearson's chisquare test to measure the differences in demographics and utilization of gynecologic procedures by the presence of psychiatric comorbidities.

\section{Results}

Psychiatric comorbidities were present in $18.7 \%$ inpatients with endometriosis. About three-fourth of these inpatients were in reproductive age group 26-45 years (75.7\%) and were whites (79.1\%). Psychiatric comorbidities were seen more in females from middle-income families and from the midwest region of the US. There was no significant difference in the utilization of gynecological procedures by the presence of psychiatric comorbidities. However, inpatients with psychiatric comorbidities had a longer mean length of stay ( 2.5 vs. 2.3 days) and total charges $(\$ 35,489$ vs. $\$ 34,673$ ) compared to the non-psychiatric cohort. Anxiety disorders predominated at $45 \%$ in patients with endometriosis followed by depressive disorder (31.3\%), psychotic disorders (12.3\%), and drug abuse (6.3\%).

Received 08/06/2020

Review began 08/11/2020 Review ended 08/16/2020 Published 08/22/2020

๑) Copyright 2020

Robert et al. This is an open access article distributed under the terms of the Creative Commons Attribution License CC-BY 4.0., which permits unrestricted use, distribution, and reproduction in any medium, provided the original author and source are credited.

\section{Conclusion}

Endometriosis with psychiatric comorbidities is prevalent in young white females from a middle-income family. Anxiety and depressive disorders are most prevalent and are associated with extended hospitalization stay and higher charges, thereby negatively impacting the healthcare burden compared to those without psychiatric comorbidities.

Categories: Obstetrics/Gynecology, Psychiatry, Quality Improvement

Keywords: nationwide inpatient sample, sociodemographic differences, depressive disorders, schizophrenia and other psychotic disorders, extended hospitalization stay, psychiatric comorbidities, endometriosis, reproductive age group

\section{Introduction}

Endometriosis is an estrogen-dependent chronic disorder characterized by the presence of endometrial type glands and stroma outside the uterus [1]. Endometriosis affects an estimated 10 million women in the United States (US), predominantly between the ages of 30 and 40 years [2].

There is an elevated risk of depression and anxiety in women with endometriosis [3-5]. This may be attributed to the consequences of endometriosis, such as chronic pelvic pain, infertility, and subfertility [6]. Endometriosis is often diagnosed later in the disease process, and this may further amplify the psychological suffering in these women and lead to psychiatric disorders [7]. Also, since both endometriosis and psychiatric disorders are heritable, familial risk factors could explain the connection [8].

Since endometriosis is often associated with a spectrum of comorbid conditions, the interactions with other comorbidities could further necessitate additional healthcare expenditure [9-11]. Explaining the role of 
psychiatric comorbidities, such as depression and anxiety, in the expenditure trajectories of endometriosis patients may aid doctors by raising awareness of the early diagnosis treatment of endometriosis.

In this study, we explore the sociodemographic differences and hospital outcomes in patients with endometriosis with versus without psychiatric comorbidities, and also evaluate the prevalence of spectrum of psychiatric disorders in these inpatients.

\section{Materials And Methods}

\section{Data source}

We conducted a cross-sectional analysis using the National Inpatient Sample (NIS, 2012 to 2014). The NIS is the largest inpatient data that consists of 4,400 hospitals across 44 states in the US. The primary and comorbid diagnostic information is identified using the International Classification of Diseases, Ninth Revision (ICD-9) and Clinical Classification Software (CCS) codes. As the NIS is a publicly available deidentified data, this study does not require approval from the institutional review board [12].

\section{Inclusion criteria and outcome variables}

We included 63,160 inpatients (age 15 to 55 years) with a primary diagnosis of endometriosis using the ICD9 codes: 617.0-617.9. The study population was further subgrouped based on codiagnosis of psychiatric comorbidities detected in the patient records using codes for attention-deficit/hyperactivity disorder (ADHD, ICD-9 codes: 314.00 or 314.01), psychotic disorders (CCS code: 659), depressive disorders (CCS code: 657), anxiety disorders (CCS code: 651), personality disorders (CCS code: 658), post-traumatic stress disorder (PTSD, ICD-9 code: 309.81), alcohol abuse (CCS code: 660 ), and drug abuse (CCS code: 661 ).

We included demographic characteristics (age, race, median household income, and region) and hospital outcomes: length of stay (LOS), i.e. the number of nights the patient was hospitalized for primary diagnosis, total charges (does not include professional fees and non-covered charges), and utilization of gynecologic procedures [13].

\section{Statistical analysis}

We used descriptive statistics and Pearson's chi-square test to measure the differences in demographics and utilization of gynecologic procedures by the presence of psychiatric comorbidities. Independent sample ttest was used for measuring the difference between mean LOS and total charges by the presence of psychiatric comorbidities. A P-value $<0.05$ was the standpoint for statistical significance in all the analyses that were done on the Statistical Package for the Social Sciences (SPSS) version 26 (IBM Corporation, Armonk, NY).

\section{Results}

We analyzed a total of 63,160 female inpatients managed for endometriosis and $18.74 \%$ had psychiatric comorbidities. About three-fourth of these inpatients with psychiatric comorbidities were in the reproductive age group 26 to 45 years (75.7\%) and were whites (79.1\%). When compared with nonpsychiatric comorbidity cohort, psychiatric comorbidities were seen more in females from families with median household income between 26 th and 50th percentile ( $27.2 \%$ vs. $25 \%$ ), and from the midwest region of the US.

There was no significant difference in the utilization of gynecological procedures between both cohorts. However, female inpatients with psychiatric comorbidities had a longer mean LOS (2.5 vs. 2.3 days) and total charges $(\$ 35,489$ vs. $\$ 34,673)$ compared to the non-psychiatric comorbidity cohort (Table 1$)$. 


\section{Cureus}

\begin{tabular}{|c|c|c|c|}
\hline \multirow{2}{*}{ Variable } & \multicolumn{2}{|c|}{ Psychiatric comorbidities, \% } & \multirow{2}{*}{ P-value } \\
\hline & $(-)$ & $(+)$ & \\
\hline Total $\mathrm{N}$ & 51325 & 11835 & - \\
\hline \multicolumn{4}{|l|}{ Age at admission } \\
\hline Mean (SD), years & $38.7(7.6)$ & $37.9(7.7)$ & $<0.001$ \\
\hline 15-25 years & 5.3 & 5.9 & \multirow{4}{*}{$<0.001$} \\
\hline 26-35 years & 27.4 & 31.4 & \\
\hline $36-45$ years & 47.0 & 44.3 & \\
\hline 46-55 years & 29.3 & 18.4 & \\
\hline \multicolumn{4}{|l|}{ Race } \\
\hline White & 63.0 & 79.1 & \multirow{4}{*}{$<0.001$} \\
\hline Black & 13.3 & 7.7 & \\
\hline Hispanic & 14.1 & 8.7 & \\
\hline Others & 9.5 & 4.6 & \\
\hline \multicolumn{4}{|l|}{ Median household income } \\
\hline 0-25th percentile & 25.2 & 24.1 & \multirow{4}{*}{$<0.001$} \\
\hline 26th-50th percentile & 25.0 & 27.2 & \\
\hline 51st - 75th percentile & 26.5 & 26.0 & \\
\hline 76th-100th percentile & 23.3 & 22.7 & \\
\hline \multicolumn{4}{|l|}{ Region } \\
\hline Northeast & 17.4 & 18.8 & \multirow{4}{*}{$<0.001$} \\
\hline Midwest & 21.3 & 25.9 & \\
\hline South & 36.8 & 31.9 & \\
\hline West & 24.5 & 23.4 & \\
\hline \multicolumn{4}{|l|}{ Hospital outcomes } \\
\hline Utilization of gynecologic procedures & 4.5 & 4.3 & 0.308 \\
\hline Mean length of stay (SD), days & $2.3(1.9)$ & $2.5(2.4)$ & $<0.001$ \\
\hline Mean total charges (SD), \$ & 34673 (28908) & 35489 (33058) & 0.005 \\
\hline
\end{tabular}

\section{TABLE 1: Distribution of endometriosis inpatients by psychiatric comorbidities}

SD: standard deviation

Out of the psychiatric comorbidities, anxiety disorders predominated at $45 \%$ in patients with endometriosis. This was followed by depressive disorder (31.3\%), psychotic disorders (12.3\%), and drug abuse (6.3\%). Other less prevalent comorbidities included ADHD (2.4\%), PTSD (1.8\%), alcohol abuse (0.6\%), and personality disorder (0.3\%) (Figure 1). 


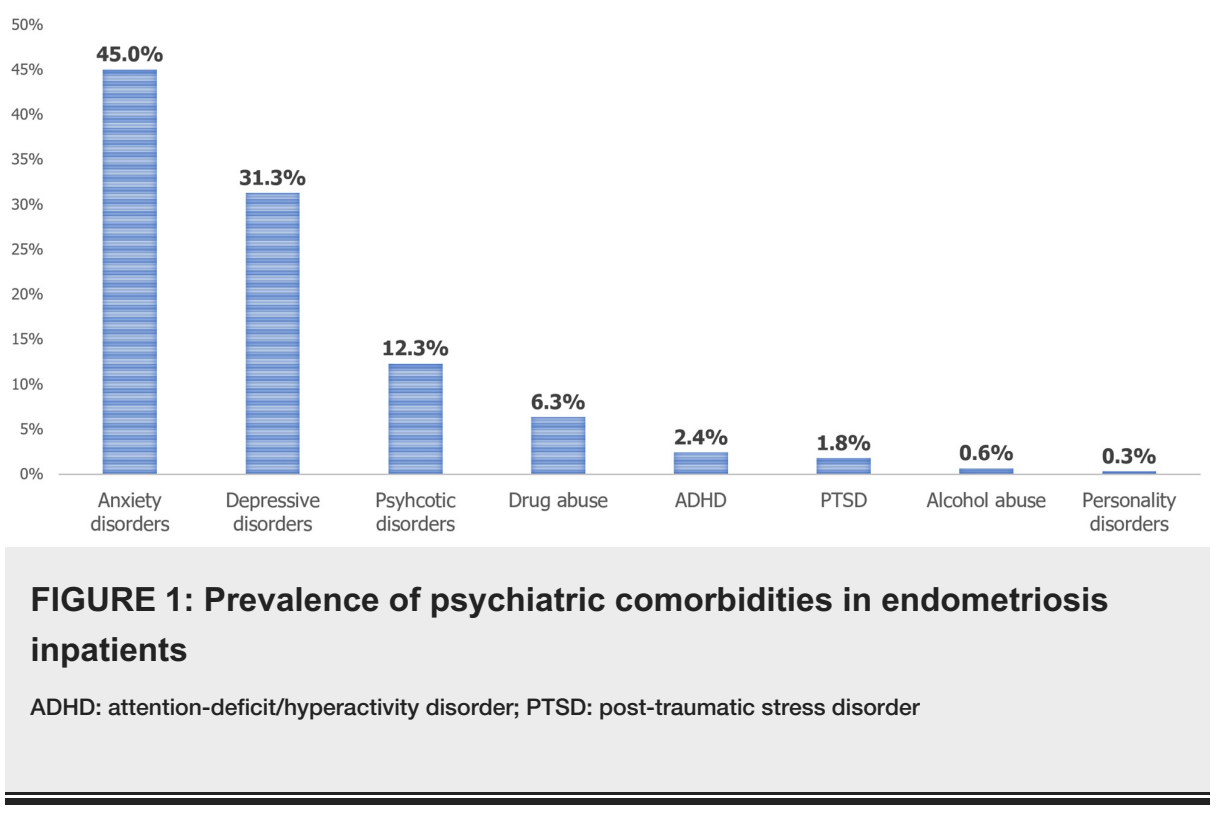

\section{Discussion}

Endometriosis has been associated with higher rates of psychiatric comorbidities, such as depression, anxiety, and poor quality of life [14]. About $29 \%$ of women with endometriosis present with moderate to severe anxiety, while depression was seen in $14.5 \%$ [15]. On the other hand, in a small sample study ( $\mathrm{N}=$ 104), it was seen that women with pelvic endometriosis had majorly have depressive symptoms (86.5\%) and anxiety (87.5\%) [4]. In our study, about $18.7 \%$ of the inpatients had psychiatric comorbidities, the most common being anxiety disorder (45\%) and depressive disorders (31.3\%). The reason for this is likely the pain and fertility complications that are symptomatic of endometriosis and chronic pelvic pain $[4,16,17]$. Moreover, endometriosis being estrogen-dependent, and treatment involves the suppression of estrogen. Therefore, treatment with oral contraceptive pills has led to side effects with decreased psychosexual arousal and adverse changes in mood, and gonadotropin-releasing hormone agonists have been found to be associated with emotional lability and depression [18].

Psychiatric comorbidities were most common in white women of reproductive age between 26 and 45 years and from lower socioeconomic status, which is in line with previous research on women with antepartum mental disorders [19]. Reproductive age in women is a time where the menstrual cycle is actively maximizing the symptoms of endometriosis given the repetitive and consistent nature of the menstrual cycle. Endometriosis has been estimated to affect $10 \%-15 \%$ of women of reproductive age [20]. The relationship between endometriosis and its impact on the patient's mental health is based on the symptoms of endometriosis, which include dyspareunia, pelvic pain, dysmenorrhea, pain with bowel movements and urination, excessive bleeding, and infertility. These symptoms often affect the psychological and social functioning of patients [4]. In our study, about four-fifth of the endometriosis inpatients were white females, which will require more future studies to identify factors inherent to the white race and related psychosocial factors.

A cross-sectional study of 59,411 women found that the incidence of endometriosis differed by geographic area, being the highest in the south (7\%) followed by the midwest (6.4\%), the west (5.4\%), and the east (5.3\%), indicating that a correlation between endometriosis and pigmentary or sun exposure may exist [21]. Our study followed the same trend with the highest incidence of endometriosis with psychiatric comorbidities in the south (31.9\%) followed by the midwest (25.9\%). This may be attributed to potential correlations between pigment characteristics, melanoma family history, and endometriosis risk [22].

The average annual incremental direct healthcare costs of endometriosis were estimated at $\$ 10,002$. In contrast, the average incremental indirect costs associated with absence hours and short-term disability compared to non-endometriosis controls were $\$ 903$ and $\$ 1,228$, respectively [23]. The incremental costs per patient in the first post-diagnosis year (over $\$ 10,000$ with multivariable adjustment) were substantial as compared to the estimates for uterine fibroids $(\$ 6,873)$ or menorrhagia $(\$ 2,878)$ in $2014[24,25]$. When associated with a spectrum of comorbid conditions, these could further necessitate additional healthcare expenditure. In our study, we found that there was statistically no significant difference in the utilization of gynecological procedures during hospitalization for endometriosis by the presence of psychiatric comorbidities. However, females with psychiatric comorbidities had significantly longer hospital LOS and higher total charges, which is in line with past inpatient and outpatient studies [19,26]. Past literature also states that psychiatric comorbidities increase the LOS for medical and surgical inpatients, in addition to gynecological inpatients, and increases medical costs leading to more systemic burden on inpatient care $[27,28]$. 
There are few limitations of this study being conducted using the administrative database as there is a lack of patient-level clinical information. Yet, the NIS is an excellent population-based representation of diseases related to systematic and temporal factors and has been used in past studies on women's mental health $[19,29]$. The major strength of this data is the large sample with national representation of the population through a uniform collection of patient records through ICD-9 codes. Since the information is coded independently by the individual practitioners, it was subject to minimal reporting bias.

\section{Conclusions}

Endometriosis with psychiatric comorbidities is prevalent in young white females from a middle-income family. Anxiety and depressive disorders are most prevalent and are associated with extended hospitalization stay and higher charges, thereby negatively impacting the healthcare burden compared to those without psychiatric comorbidities. These comorbidities may worsen the overall symptomatology and disease severity of endometriosis that adversely impacts their relationships, quality of life, and functionality. Early diagnosis and treatment of at-risk patients with psychiatric comorbidities should be done by implementing routine mental health screening for patients with endometriosis to improve their healthrelated quality of life. Such action from the clinicians and healthcare systems has the potential to improve the mental health of women with endometriosis thereby promoting success in educational, interpersonal, and occupational aspects of life. Future studies should find the prevalence of psychiatric comorbidities in patients diagnosed with endometriosis earlier versus later in life, and the effect that the time of diagnosis has on the severity of illness, hospital outcomes, and the utilization of gynecological procedures.

\section{Additional Information}

\section{Disclosures}

Human subjects: Consent was obtained by all participants in this study. Animal subjects: All authors have confirmed that this study did not involve animal subjects or tissue. Conflicts of interest: In compliance with the ICMJE uniform disclosure form, all authors declare the following: Payment/services info: All authors have declared that no financial support was received from any organization for the submitted work. Financial relationships: All authors have declared that they have no financial relationships at present or within the previous three years with any organizations that might have an interest in the submitted work. Other relationships: All authors have declared that there are no other relationships or activities that could appear to have influenced the submitted work.

\section{References}

1. Burney RO, Giudice LC: Pathogenesis and pathophysiology of endometriosis. Fertil Steril. 2012, 98:511-519. 10.1016/j.fertnstert.2012.06.029

2. Endometriosis. (2020). Accessed: June 5, 2020: https://www.endtoendo.com/Endometriosis_Overview_End_to_Endometriosis.html.

3. Pope CJ, Sharma V, Sharma S, Mazmanian D: A systematic review of the association between psychiatric disturbances and endometriosis. J Obstet Gynaecol Can. 2015, 37:1006-1015. 10.1016/s1701-2163(16)300500

4. Lagana AS, La Rosa VL, Rapisarda AMC, et al.: Anxiety and depression in patients with endometriosis: impact and management challenges. Int J Womens Health. 2017, 9:323-330. 10.2147/IJWH.S119729

5. Cavaggioni G, Lia C, Resta S, Antonielli T, Benedetti Panici P, Megiorni F, Porpora MG: Are mood and anxiety disorders and alexithymia associated with endometriosis? a preliminary study. Biomed Res Int. 2014, 2014:786830.

6. Gao M, Koupil I, Sjoqvist H, Karlsson H, Lalitkumar S, Dalman C, Kosidou K: Psychiatric comorbidity among women with endometriosis: nationwide cohort study in Sweden [Epub ahead of print]. Am J Obstet Gynecol. 2020, 10.1016/j.ajog.2020.02.033

7. Kuznetsov L, Dworzynski K, Davies M, Overton C, Guideline Committee: Diagnosis and management of endometriosis: summary of nice guidance. BMJ. 2017, 358:j3935. 10.1136/bmj.j3935

8. Cross-Disorder Group of the Psychiatric Genomics Consortium, Lee SH, Ripke S, et al.: Genetic relationship between five psychiatric disorders estimated from genome-wide SNPs. Nat Genet. 2013, 45:984-994. 10.1038/ng.2711

9. Simoens S, Dunselman G, Dirksen C, et al.: The burden of endometriosis: costs and quality of life of women with endometriosis and treated in referral centres. Hum Reprod. 2012, 27:1292-1299. 10.1093/humrep/des073

10. Soliman AM, Yang H, Du EX, Kelley C, Winkel C: The direct and indirect costs associated with endometriosis: a systematic literature review. Hum Reprod. 2016, 31:712-722. 10.1093/humrep/dev335

11. Fuldeore M, Yang H, Du EX, Soliman AM, Wu EQ, Winkel C: Healthcare utilization and costs in women diagnosed with endometriosis before and after diagnosis: a longitudinal analysis of claims databases. Fertil Steril. 2015, 103:163-171. 10.1016/j.fertnstert.2014.10.011

12. Overview of the National (Nationwide) Inpatient Sample . (2019). Accessed: March 15, 2020: https://www.hcup-us.ahrq.gov/nisoverview.jsp.

13. NIS description of data elements . (2019). Accessed: March 15, 2020: https://www.hcupus.ahrq.gov/db/nation/nis/nisdde.jsp.

14. Smorgick N, Marsh CA, As-Sanie S, Smith YR, Quint EH: Prevalence of pain syndromes, mood conditions, and asthma in adolescents and young women with endometriosis. J Pediatr Adolesc Gynecol. 2013, 26:171175. 10.1016/j.jpag.2012.12.006 
15. Friedl F, Riedl D, Fessler S, et al.: Impact of endometriosis on quality of life, anxiety, and depression: an austrian perspective. Arch Gynecol Obstet. 2015, 292:1393-1399. 10.1007/s00404-015-3789-8

16. Vannuccini S, Lazzeri L, Orlandini C, Morgante G, Bifulco G, Fagiolini A, Petraglia F: Mental health, pain symptoms and systemic comorbidities in women with endometriosis: a cross-sectional study. J Psychosom Obstet Gynaecol. 2018, 39:315-320. 10.1080/0167482X.2017.1386171

17. Lorencatto C, Petta CA, Navarro MJ, Bahamondes L, Matos A: Depression in women with endometriosis with and without chronic pelvic pain. Acta Obstet Gynecol Scand. 2006, 85:88-92. 10.1080/00016340500456118

18. Sanders SA, Graham CA, Bass JL, Bancroft J: A prospective study of the effects of oral contraceptives on sexuality and well-being and their relationship to discontinuation. Contraception. 2001, 64:51-58. 10.1016/s0010-7824(01)00218-9

19. Bhimanadham NN, Mainali P, Robert CA, Masroor A, Onyeaka HK, Hossain S, Patel RS: Hospital outcomes in antepartum mental disorders: a study on 897,397 pregnant inpatients. Behav Sci. 2019, 9:105. 10.3390/bs9100105

20. Olive DL, Pritts EA: Treatment of endometriosis. N Engl J Med. 2001, 345:266-275. 10.1056/NEJM200107263450407

21. Fuldeore MJ, Soliman AM: Prevalence and symptomatic burden of diagnosed endometriosis in the United States: national estimates from a cross-sectional survey of 59,411 women. Gynecol Obstet Invest. 2017, 82:453-461. 10.1159/000452660

22. Kvaskoff M, Han J, Qureshi AA, Missmer SA: Pigmentary traits, family history of melanoma and the risk of endometriosis: a cohort study of US women. Int J Epidemiol. 2014, 43:255-263. 10.1093/ije/dyt235

23. Soliman AM, Surrey E, Bonafede M, Nelson JK, Castelli-Haley J: Real-world evaluation of direct and indirect economic burden among endometriosis patients in the United States. Adv Ther. 2018, 35:408-423. 10.1007/s12325-018-0667-3

24. Fuldeore M, Yang H, Soliman AM, Winkel C: Healthcare utilization and costs among women diagnosed with uterine fibroids: a longitudinal evaluation for 5 years pre- and post-diagnosis. Curr Med Res Opin. 2015, 31:1719-1731. 10.1185/03007995.2015.1069738

25. Jensen JT, Lefebvre P, Laliberte F, Sarda SP, Law A, Pocoski J, Duh MS: Cost burden and treatment patterns associated with management of heavy menstrual bleeding. J Womens Health. 2012, 21:539-547. 10.1089/jwh.2011.3147

26. Mendonsa RD, Appaya P: Psychiatric morbidity in outpatients of gynecological oncology clinic in a tertiary care hospital. Indian J Psychiatry. 2010, 52:327-332. 10.4103/0019-5545.74307

27. Wancata J, Benda N, Meise U, Windhaber J: Does psychiatric comorbidity increase length of stay in medical, surgical and gynecological departments? (Article in German). Nervenarzt. 1999, 70:810-816. 10.1007/s001150050516

28. Wolff J, Heister T, Normann C, Kaier K: Hospital costs associated with psychiatric comorbidities: a retrospective study. BMC Health Serv Res. 2018, 18:67. 10.1186/s12913-018-2892-5

29. Ahmad N, Robert CA, Jampa A, Ashraf S, Patel RS: Antepartum drug dependence and pregnancy- or birthrelated complications: a cross-sectional study of 19 million inpatients. Cureus. 2019, 11:e6117. 10.7759/cureus.6117 